Regine Witkowski

Falko H. Herrmann

\title{
Einführung \\ in die klinische Genetik
}




\section{REIHE WISSENSCHAFT}

Die REIHE W ISSE NSCHAFT ist die wissenschaftliche Handbibliothek des Naturwissenschaftlers und Ingenieurs und des Studenten der mathematischen, naturwissenschaftlichen und technischen Fächer. Sie informiert in zusammenfassenden Darstellungen über den aktuellen Forschungsstand in den exakten Wissenschaften und erschließt dem Spezialisten den Zugang zu den Nachbardisziplinen. 
Regine Witkowski

Falko H. Herrmann

\section{Einführung in die klinische Genetik}

Mit 35 Abbildungen

und 6 Tabellen

$\mathbf{v}$

Vieweg · Braunschweig 
Verantwortlicher Herausgeber dieses Bandes:

Prof. Dr. E. Hofmann, Leipzig

Verfasser:

Dr. habil. Regine Witkowski

Dr. sc. nat. Falko H. Herrmann

Berlin/Halle

1976

Alle Rechte vorbehalten

(C) Akademie-Verlag, Berlin, 1976

Softcover reprint of the hardcover 1st edition 1976

Lizenzausgabe für

Friedr. Vieweg \& Sohn Verlagsgesellschaft mbH, Braunschweig mit Genehmigung des Akademie-Verlages, DDR-Berlin.

ISBN 978-3-528-06819-6

DOI 10.1007/978-3-322-85525-1

ISBN 978-3-322-85525-1 (eBook) 


\section{Vorwort}

Die Fortschritte der Medizin in den letzten Jahrzehnten, insbesondere auf dem Gebiet der Bekämpfung der Neugeborenen- und Kindersterblichkeit haben den Kreis der letal endenden oder mit Dauerschäden einhergehenden Krankheiten des Menschen stark eingeengt. Dadurch nehmen innerhalb der verbliebenen Gruppe die nicht oder nur ausnahmsweise therapierbaren Erbleiden an Bedeutung zu. Von diesem Gesichtspunkt ausgehend soll das Anliegen dieses Taschenbuches ein zweifaches sein: Es soll, aufbauend auf genetischen und biochemischen Grundkenntnissen, in die wichtigsten Erkenntnisse der medizinischen Genetik einführen und deren Bedeutung und Möglichkeiten für die medizinische Praxis erläutern und es soll gleichzeitig eine breitere Einführung in das Wörterbuch für die Familienberatung (Genetik erblicher Syndrome und Mißbildungen, Gustav Fischer Verlag, Stuttgart 1976) darstellen, wie sie von vielen Lesern für wünschenswert gehalten wurde. Aus Gründen der Übersichtlichkeit haben wir im Text erwähnte Krankheitsbilder nicht näher beschrieben. Eine Charakterisierung der wichtigsten von ihnen wurde im Anhang zusammengestellt, so daß eine Orientierung möglich ist.

Dem Charakter als Taschenbuch entsprechend wird nur bedingt auf zugrunde liegende Literatur eingegangen, eine Aufstellung zusammenfassender genetischer Darstellungen ist für den interessierten Leser angefügt.

Die Verfasser 


\section{Inhalt}

1. Die Mendelschen Gesetze . . . . . . . . . 9

1.1. Die Universalität des genetischen Codes und genetischer Gesetzmäßigkeiten . . . . . . . 9

1.2. Die Mendelschen Gesetze in ihrer Anwendung auf den Menschen . . . . . . . . . . . . . 10

1.2.1. Erstes Mendelsches Gesetz: Uniformitätsregel. 10

1.2.1.1. Homozygotie und Heterozygotie . . . . . . . 10

1.2.1.2. Dominanz und Rezessivität . . . . . . . . . 12

1.2.1.3. Homo- und Heterogenie . . . . . . . . . . . . 16

1.2.1.4. Polygenie . . . . . . . . . . . . . . . . . 21

1.2.2. Zweites Mendelsches Gesetz: Spaltungsregel . 22

1.2.2.1. Grenzen und Möglichkeiten erbprognostischer Aussagen auf Grund des zweiten Mendelschen Gesetzes . . . . . . . . . . . . . 23

1.2.2.2. Kodominanz und intermediäre Vererbung . . . 25

1.2.2.3. Unvollständig dominante oder inkomplett rezessive Vererbung . . . . . . . . . . . . . 26

1.2.2.3.1. Merkmalsfreie Anlagenträger . . . . . . . . . 27

1.2.2.3.2. Intrafamiliäre Variabilität, variable Expressivität . . . . . . . . . . . . . . . . . 28

1.2.2.3.3. Penetranz und Expressivität . . . . . . . . . 28

1.2.2.4. Spaltungsverhältnisse und Risikoziffern bei Polygenie . . . . . . . . . . . . . . . . . . 30

1.2.3. Drittes Mendelsches Gesetz: Regel von der Neukombination der Gene . . . . . . . . . . . . 30

1.2.3.1. Kopplung, Kopplungsgruppen . . . . . . . . 31

1.2.3.2. Die Chromosomen des Menschen . . . . . . . 32

1.2.3.3. Methoden zur Feststellung von Kopplungsgruppen und zur Genlokalisation . . . . . . . . . 34

1.2.3.3.1. Stammbaumuntersuchungen . . . . . . . . . 34

1.2.3.3.2. Geschlechtsgekoppelte Vererbung . . . . . . 36

1.2.3.3.2.1. Kopplungsgruppe Y-Chromosom . . . . . . . 36 
1.2.3.3.2.2. Kopplungsgruppe $\mathrm{X}$-Chromosom, X-chromosomale Vererbung

1.2.3.3.3. Direkte Genlokalisierung mit Hilfe von Chromosomenaberrationen . . . . . . . . . . . . . 43

1.2.3.3.4. Genlokalisierung mit Hilfe der Zellhybridisation 44

1.2.3.3.5. Nukleinsäure-Hybridisierung . . . . . . . . . 45

1.2.3.4. Kopplung und freie Kombinierbarkeit der Gene. 45

2. Mutationen. . . . . . . . . . . . . 46

2.1. Genommutationen beim Menschen . . . . . . 46

2.1.1. Polyploidie. . . . . . . . . . . . . . . 46

2.1.2. Aneuploidie . . . . . . . . . . . . . . . . 47

2.1.2.1. Aneuploidien der Geschlechtschromosomen . . . 48

2.1.2.2. Aneuploidien der Autosomen . . . . . . . . . 50

2.1.2.3. Entstehungsweise von Aneuploidien; Nondisjunction . . . . . . . . . . . . . . . . . . . . 52

2.1.2.4. Aneuploidie und Lebensalter . . . . . . . . . 55

2.1.3. Chromosomale Veränderungen beim Krebs . . . 58

2.2. Chromosomenmutationen . . . . . . . . . 60

2.2.1. Chromosomenaberrationstypen beim Menschen . 60

2.2.2. Deletionen, Defizienzen . . . . . . . . . . 61

2.2.2.1. Deletions-Syndrome . . . . . . . . . . . . . 61

2.2.2.2. Merkmalsausprägung bei partieller Monosomie . 61

2.2.2.3. Ringchromosomen . . . . . . . . . . . . . 64

2.2.2.4. Isochromosomen . . . . . . . . . . . . . . 64

2.2.3. Translokationen . . . . . . . . . . . . . . 66

2.2.3.1. Reziproke Translokationen und ihre Konsequenzen . . . . . . . . . . . . . . . . . . . . 66

2.2.3.2. Translokationstrisomie beim Down-Syndrom funktionelle Trisomie durch zentrische Fusion . 68

2.2.3.3. Erbprognose beim Down-Syndrom . . . . . . 71

2.2.3.4. Unbalancierte Translokationen - erbliche partielle Monosomien oder Trisomien . . . . . . . 73

2.2.4. Inversionen . . . . . . . . . . . . . . . . 74

2.2.5. Erhöhte Chromosomenbrüchigkeit . . . . . . 74

2.3. Genmutationen . . . . . . . . . . . . . . . 76

2.3.1. Verschiedene Typen der Genmutationen und ihre phänotypischen Konsequenzen . . . . . . . . 76

2.3.2. Mutative Veränderungen an Strukturproteinen - die Hämoglobinvarianten . . . . . . . . . 79

2.3.3. Mutative Veränderungen an Enzymproteinen . 83

2.3.3.1. Enzymvarianten (Isozyme der G6PD) . . . . . 83 
2.3.3.2. Konsequenzen der Aminosäuresubstitution für die Funktion der Polypeptide . . . . . . . . . . 85

2.3.3.3. Inborn errors of metabolism - angeborene Stoffwechselkrankheiten . . . . . . . . . . . . . 86

2.3.4. Genmutation und Phänotyp . . . . . . . . . 89

2.3.4.1. Polymorphismen . . . . . . . . . . . . . . 89

2.3.4.2. Selektionsvorteil Heterozygoter . . . . . . . 92

2.3.4.3. Multiple Allelie . . . . . . . . . . . . . . . . . 92

2.3.4.4. Heterogenie . . . . . . . . . . . . . . . . 95

2.3.5. Entstehung von Genmutationen . . . . . . . 98

2.3.5.1. Wirkungsweise mutagener Faktoren . . . . . . 99

2.3.5.1.1. Chemikalien und Strahlen . . . . . . . . . . 99

2.3.5.1.2. Viren . . . . . . . . . . . . . . . . 101

2.3.5.2. Die häufigsten Schadenstypen in der DNS . . . 101

2.3.6. Genmutationen beim Menschen in Soma- und Keimzellen . . . . . . . . . . . . . 105

2.3.6.1. Mutationshäufigkeit in Abhängigkeit vom Lebensalter . . . . . . . . . . . . . . 107

2.3.6.2. Somatische Mutationen . . . . . . . . . . . 109

2.3.6.3. Mutationen im Reparatursystem des Menschen Xeroderma pigmentosum . . . . . . . . 110

3. Populationsgenetik . . . . . . . . . . . . . 112

3.1. Frequenz und Inzidenz . . . . . . . . . . . 113

3.1.1. Frequenz . . . . . . . . . . . . . . . 113

3.1.2. Inzidenz . . . . . . . . . . . . . . . . . . 113

3.2. Häufigkeit dominanter Allele . . . . . . . . 114

3.3. Häufigkeit rezessiver Gene - das Hardy-WeINBERGsche Gesetz . . . . . . . . . . . . . . 116

3.4. Genetische Bürde und Eugenik . . . . . . . . 119

3.4.1. Gleichgewicht zwischen Selektion und Neumutation. . . . . . . . . . . . . . . . 125

3.4.2. Erbleiden mit relativ hoher Frequenz . . . . . 127

3.5. Genetisch bedingte Defekte und Umweltfaktoren

- Zwillingsforschung . . . . . . . . . . 128

4. Die genetische Familienberatung . . . . . 131

4.1. Aufgaben der genetischen Familienberatung . . 131

4.2. Die Familienanamnese . . . . . . . . . . . 132

4.3. Das Problem der Verwandtenehen . . . . . . 137

4.4. Klinisch-genetische Diagnostik . . . . . . . . 139

4.4.1. Suche nach Mikro- und Teilsymptomen . . . 139 
4.4.2. Heterozygotentest. . . . . . . . . . . . . 140

4.4.3. Screening-Teste . . . . . . . . . . . . . . . 152

4.5. Die individuelle Beratung . . . . . . . . . . 155

4.5.1. Einschätzung des Risikos - Therapie und Pro-

gnose . . . . . . . . . . . . . . . . 155

4.5.2. Die pränatale Diagnostik. . . . . . . . . . . 158

4.5.3. Multifaktoriell bedingte Krankheitsbilder. . . . 166

5. Schlußwort ............... 171

6. Anhang ............... . 173

7. Literatur. . . . . . . . . . . . 206

8. Sachregister . . . . . . . . . . . . 209 